\title{
Postintensive care syndrome-family in Intensive Care Units: "What is it in the name?" A Scoping Definitive Review
}

\author{
Frank Kiwanuka ${ }^{1 *}$ and Sanaz Akhavan $\operatorname{Rad}^{2}$ \\ ${ }^{1}$ Department of Critical Care Nursing, Tehran University of Medical Sciences-International Campus, Iran \\ ${ }_{2}^{2}$ Indepedent Researcher, Iran
}

*Corresponding author: Frank Kiwanuka, Department of Critical Care

Nursing, Tehran University of Medical Sciences-International Campus, Iran.

Received Date: December 20, 2018

Published Date: March 01, 2019

\begin{abstract}
Aim: In this review, we sought to succinctly review what Postintensive care syndrome is, it's risk factors, consequences, assessment, diagnosis, prevention and treatment among family members of intensive care patients.

Background: The proportion of family members caring for a patient in intensive care units is expected to proportionally increase with increase in utilization of intensive care units due to various reasons. Subsequently, unfavorable physical, psychosocial responses due to exposure to complications following admission of a family member in intensive care due to critical illness will consequently increase among family members. These unfavorable family responses to critical illness have been termed "Postintensive care syndrome-family (PICS-F)." Postintensive care syndrome-family encompasses: anxiety, acute stress disorder, post-traumatic stress, depression and complicated grief. Healthcare providers as well as family members need to be knowledgeable on the extent, diagnosis, prevention and outcomes of these sequelae. Relationship to the patient, younger patients, prior experience of ICU, low economic status, length of ICU stay, unmet self-care, communication needs and distance from the hospital seem to predispose family members to PICS-F. Longer distance from hospital and higher resilience seem to protect family members from PICS-F. Prevention of PICS-F includes: effective family-ICU staff communication, enhancing resilience and coping, post discharge planning and follow-up interventions.
\end{abstract}

Conclusion: results highlight the importance of acknowledging experiences of family members having a patient admitted in intensive care unit. Healthcare professionals need to have insights into this phenomenon and optimally intervene to prevent these physiological and psychosocial sequelae.

Keywords: Postintensive care syndrome family; Family; Intensive care; Critical care; Anxiety; Posttraumatic stress disorder; Depression

Abbreviations: ICU: Intensive Care Unit; PICS: Post Intensive Care Syndrome; PICS-F: Post Intensive Care Syndrome- Family

\section{Introduction}

Health and illness are family events therefore; an individual family member's health affects the entire family functioning. A family can simply be defined as "who the member says it is," implying that, it is not limited to people with ancestral linkages. In context, the family holds strong health practices, attitude and beliefs and interrelationships [1]. Why, however should we be concerned about family members of patients once admitted in ICU? Healthcare providers need to be concerned about the family members in the context of Intensive Care owing to advances in intensive care and the increasing graying population, there is increasing utilization of intensive care services. Eventually, the proportion of family caregivers and families having experiences of Intensive Care Units (ICU) is consequently growing [2].

Experience of a family member in ICU has long standing compilations which may present up to more than four year following discharge from ICU [3]. As a result, nurses and other healthcare 
providers need to be knowledgeable, intervene, promote health, identify, prevent illness risks and rehabilitate those experiencing complications. The Society of Critical Care Medicine has termed this myriad of complications as "Postintensive care syndromefamily" (PICS-F). Consequently, PICS-F has negative implications on the patient and family following discharge from ICU [4-6]. There is a growing body of literature on psychological experiences of relatives of patients admitted in ICU globally. This could partly be attributed to the growing number of patients admitted in ICU and ICU survivors. In addition, studies have reported remarkable risk of depression, anxiety and posttraumatic disorders among family members of patients admitted in ICU. There are previous reviews that have attempted to discuss psychological repercussions in family members of ICU patients.

For Instance: Jezierska [5] and colleagues explored the psychological repercussions and prevention measures to PICS-F. A similar narrative by Schmidt \& Azoulay [7] also appraised the significance of PICS-F burden among family members, its prevention measures and consequences for the family. Another review by Davidson et al. [3] also highlighted the epidemiology, assessment and interventions. The above reviews present seminal evidence on PICS-F however; in depth evidence on interventions to prevent PICS-F is unremarkable. In this scoping review, we succinctly describe PICS-F among family members of adult intensive care patients including: what it is, risk factors, consequences, assessment, diagnosis, treatment and prevention measures. Scoping reviews are important in expeditiously mapping out literature on a topic. They are methodologically more exploratory which permits broader evidentiary understanding that can inform the development of research questions that guide interventions, systematic reviews and empirical studies [8,9].

\section{Discussion}

\section{What is it in a name "PICS-F?"}

There has been remarkable progress in terms of survival from critical illness and increased utilization of intensive care units. However, this is not without repercussions for the family members of critically ill patients ever admitted to ICU. Family member often suffer significant impairment termed Postintensive Care Syndrome-Family. Post intensive care syndrome-family depicts a cluster of psychological complications among relatives of patients admitted in ICU [6]. Postintensive care syndrome-family involves development of adverse psychological and physical outcomes including anxiety, acute stress disorder, post-traumatic stress disorder (PTSD), depression and complicated grief $[3,7,10]$.

The prevalence of these psychological problems among family members is remarkable and varies across studies globally. Noteworthy, anxiety is the commonest psychological disorder in the PICS-F cluster experienced by family members. Anxiety has been reported among $81.4 \%$ of family members, while depression is experienced in up to $94.2 \%$ of family members of ICU patients [11]. A similar study of Kose et al. [12] showed that $35.9 \%$ and $71.8 \%$ of family members experienced anxiety and depression respectively. Another study showed that $45 \%, 18 \%$ and $40 \%$ of family members of patients admitted in ICU had symptoms of anxiety, depression and acute stress respectively [13]. A similar study in Czech and Slovak Republics showed that a high proportion (78\% and 54\%) of family members of patients in ICU experienced anxiety and depression respectively [14], another study in the Netherlands revealed that $15 \%$ family members of previously ICU patients experienced PTSD [15]. These statistics remarkably illustrate the fact that family members experience significant consequences from the time of admission of a family member to discharge and beyond.

\section{Risk Factors}

Various studies have revealed different risk factors for PICS-F. These have been largely studied depending on the component of PICS-F under consideration. Celik et al. [11] showed that married, low income and family members who used regular medication experienced significantly higher scores of anxiety and depression. A positive association was also liked to age, and length of hospital stay. Some studies have highlighted that family members of patients with shorter length of stay ( 2 days) have similar prevalence of anxiety and depression at discharge as those with longer length of stay [16]. The association of length of stay and PICS-F could be attributed to onset of worries among family members. However, this phenomenon has not yet been significantly assessed in a research setting. Other situations which were reported to be associated with more experiences of anxiety and depression included: when no other person accompanied them, unmet self-care needs [11,13] and unmet information needs [11]. In this regard, the association of marriage and experiences of anxiety and depression among family members could be attributed to the closeness of the relationship inform of a husband and wife [12]. The closeness of the relationship could point to the commitment to care of family members and friends in need of assistance [17], as such this could trigger depression and anxiety due uncertainty about outcomes of critical illness. Regarding economic status, better economic statuses are associated with more happiness and standards of living which usually but not always minimize stress [18]. Age of both the patient and the family member: Having a younger patient admitted in ICU has also been reported to be associated with family members who have younger patients admitted in ICU [12].

Family-ICU staff communication; limited family-ICU staff communication has also been associated with higher experiences of anxiety and depression [14]. History of familial or personal experience of intensive care has also been associated with higher prevalence of psychological distress among family members. Lewis and Taylor [19] pointed out that ICU experience in the past two years is significantly more associated with depression and acute stress. Family members' previous experience in ICU may contribute to responses of the current situation especially if the experience was unfavorable could lead to increased anxiety, depression and acute stress disorder symptoms in a subsequent admission.

\section{Protective factors}

Increasing distance from the family members' home has been associated with reduction in anxiety and depression among family members of patients in ICU [11]. Resilience has also been shown to be associated with reduced experiences of anxiety, depression and acute stress among family members of patients in ICU. More 
resilient family members experience relatively lower rates of anxiety and depression compared to their counterparts with low resilience [20].

\section{Consequences of PICS-F}

PICS-F often has intense psychological and physical repercussions for family members of patients admitted in ICU. The detrimental effects may persist following year after discharge from ICU. Anxiety and depression among family members of patients admitted in ICU has been reported to be associated with sleep problems [11,21,22]. Other symptoms such as cardiac anxiety [23] have also been isolated to be specific to family members experiencing PICS-F. Cardiac anxiety is a specific heart-focused anxiety (HFA) characterized by fear of cardiac-related events and sensations arising from perceived undesirable consequences [24].

Overburden among family members is not uncommon following discharge from ICU. Discharge often leaves family members at the cross road on how to navigate the responsibilities of balancing personal needs, the continuum of care for the patient and work resumption. Wolters' study in the Netherland revealed that $23 \%$ of family members of patients experienced overburden following discharge. Furthermore, the ICU experience has profound effect on work resumption with many family members being able to resume work following months [15]. This provides insights in economic burden that family members have following an experience of critical illness or injury. Lastly, complicated grief is not uncommon among family members. This could be attributed to the fact that up to $50 \%$ of deaths in the first year following ICU admission occur after discharge [25]. This presents a complicated situation for the family which had already experienced a set of circumstances during ICU journey.

\section{Assessment and diagnosis}

In practice, a comprehensive examination notwithstanding experiences of a relative with critical illness could raise a high suspicion index which could be seminal in identification of PICS-F. In addition, a number of remarkable tools have been developed to assess each of the components of PICS-F. Validated tools that can be used to identify symptoms pointing to anxiety, depression, PTSD and complicated grief include but not limited to the following: the modified mini-mental state examination (MMSE) [26], the MiniCog, the Montreal Cognitive Assessment (MoCA), Hospital anxiety and depression scale, Beck Depression Inventory [27], Beck Anxiety Inventory [28,29], Posttraumatic stress syndrome 10-questions inventory (PTSS-10), the Zung depression and anxiety scales [3032], the patient health questionnaire-2 and -9 forms, Posttraumatic Stress Disorder checklist-Event Specific Version (PCL-S), item mapping according to the Diagnostic and Statistical Manual of Mental Disorders-IV. We recommend that the choice of tool should be consistent with the user's familiarity, effectiveness of the tool and item content.

\section{Prevention measures and treatment}

Various Evidence based interventions addressing PICS-F have been presented in a number of remarkable studies. Notably, most interventions have revolved around improving the quality and extent of family-ICU staff communication. Firstly, communication should be clear, reciprocal and delivered frequently. Various interventions of delivering effective communication have been studied in several substantive studies. For instance: Locke et al. [10] explored the feasibility and sustainability of an ICU diary program using Iowa Model of Evidence-Based Practice. Their intervention involved nurse-initiated diaries on patients who were intubated for more than 24 hours.

The diary was used as a record of the reason warranting ICU admission and a continuum of shift-based entries by nurses, physicians and other healthcare providers. The outcomes of the project showed that it helped patients and their family to cope with critical illness [10]. Hence, a daily record and updating family could afford mitigating undesirable ICU outcomes. Other studies have studied the feasibility and effectiveness of information brochures, websites and have revealed positive results regarding reduction of one or more components of PICS-F [33,34].

Family and patient education: Importantly, we recommend education across the recovery continuum. In fact, educating the family on the consequences of critical illness could be beneficial to the family. The first hint is entailed in educating them on the magnitude of debilitation the patient has experienced. Awareness of the generalized body weakness could create an understanding of the systematic muscle injury and loss that occurs as a result of critical illness of the patient. This could prevent the physical and psychological stress experienced after discharge when the patient is surprised to learn that they cannot do the same things they used to before the injury, the long time to recover and the need for family members to support them in doing some activities.

Enhancing resilience and coping: Some interventions have used cognitive based models to enhance resilience among family members. These are aimed at enhancing the family members' ability to deal with their situation of having a love one being critically ill and hospitalized in ICU. Chiang and colleagues showed that cognitive-behavioral psycho-education enhanced family members' ability to cope with stress associated with the ICU experience [35]. Similar studies have also showed that enhancing resilience could afford reducing stress among family members $[13,20]$. Screening: assessing for known predisposing factors in ICU family members and identification of those that could benefit from support services could go a long way in preventing precedential psychological sequelae among ICU family members. Awareness of family members' previous experience in ICU may contribute to increased anxiety, depression and acute stress disorder symptoms in a subsequent admission may choose to approach these family members with greater sensitivity and awareness of their psychological wellbeing during the hospital stay [19].

\section{Informed skilled and professionals}

Kean and Smith [35] posited that "no ICU patient survives if there is not skilled and professional nursing care that goes with those medical advances. For in reality, surviving a critical illness does come at a cost for patients and their families and nurses care at different times during this journey". Indeed, nurses, physicians and other healthcare providers need to be aware of scope and 
practices of intensive care and the continuum of care beyond discharge. Following discharge focus is shifted to the complexity of Postintensive sequelae and challenges faced by families. In this context, healthcare professionals need to be knowledgeable and skilled on how to rebuild the family and the patient's life after intensive care. Noteworthy, rehabilitation could go a long way in re-engaging the family and patient in their previous social life. This could enable overall physical and psychological recovery.

\section{Post-ICU care}

ICU recovery is often a long-term process depending on the extent of deficits imposed by the disease or injuries. Post-ICU care can be done during follow-up visits after discharge interventions, after ICU clinics and rehabilitation programs. It is moral reasoning that family members experience psychosocial and financial consequences after discharge from ICU. Therefore, it stands to reason that they require support following discharge from ICU. Post-ICU Care addresses the long-term complications of PICS-F. Huggins et al. [4] reported on an Interprofessional post-ICU clinic at Vanderbilt University Medical Center which provided survivors and families with care after discharge. In furtherance, ICU bereavement services such sending sympathy cards to the family, bereavement follow-up services and follow-up calls to families have been reported to be remarkable interventions for families. They have been widely utilized in countries such as Australia and New [36].

\section{Evidence}

Lastly, we prescribe more research into this domain. Policy driven change leaves many gaps if it is not informed by credible research. Families in ICU generally constitute an underscored research group. Going further, the drive towards patient and family centered care approaches that advocate for more involvement of the family in care, needs research insights including: relative importance of interventions, magnitude of effects, precision of the estimates of effects and cost. While this arena is starting to register a significant number of remarkable randomized trials [33,37], qualitative studies would also go a long way in informing policy on the experiences of families during and following ICU discharge. Quantitative and mixed methods studies cannot be underscored in reporting the prevalence and assessing a wide array of associated factors to PICS-F. Such evidence could provide clinicians, policy makers and families with evidence-based strategies to optimize support given to families of critically ill patients in ICU during and following discharge in attempt of preventing PICS-F.

\section{Conclusions and implications for practice}

Family members of ICU patients experience one or more of the disorders that form a cluster of psychological disorders following admission of a patient to ICU. Nurses and other frontline clinicians need to acknowledge the significance of experiences of family members following admission of a patient in ICU and after discharge. Consumer assessment of healthcare providers and systems should encompass evaluation of psychological ICU experiences among family members of patients admitted in ICU. This review presented what PICS-F is and prevalence of the cluster of psychological disorders that form PICS-F, risk factors, consequences and prevention measures. It informs healthcare professional, family members, policy and practice on the extent, need to address the problem and strategies that could be used to prevent adverse psychological sequelae that follow ICU admission.

\section{Acknowledgement}

We are grateful for the support of Mr. Waliu Jawula Salisu and Mr. Yakubu Nichlas Dayah for the tremendous recommendations accorded to this article.

\section{Conflict of Interest}

No Conflict of Interest.

\section{References}

1. Kaakinen JR, Coehlo DP, Steele R, Robinson M, FA Davis (2018) Family health care nursing: Theory, practice, and research.

2. Maguire JM, Carson SS (2013) Strategies to combat chronic critical illness: Curr Opin Crit Care 19(5): 480-487.

3. Davidson JE, Jones C, Bienvenu OJ (2012) Family response to critical illness: Postintensive care syndrome-family. Critical care med 40(2): 618-624.

4. Huggins EL, Bloom SL, Stollings JL, Camp M, Sevin CM, et al. (2016) A Clinic Model: Post-Intensive Care Syndrome and Post-Intensive Care Syndrome-Family. AACN Adv Crit Care 27(2): 204-211.

5. Jezierska N (2014) Psychological reactions in family members of patients hospitalized in intensive care units. Anaesthesiol Intensive Ther 46(1): 42-45.

6. (2013) Society of Critical Care Medicine. Post-Intensive Care Syndrome.

7. Schmidt M, Azoulay E (2012) Having a loved one in the ICU: the forgotten family. Curr Opin Crit Care 18(5): 540-547.

8. Arksey H, O Malley L (2005) Scoping studies: towards a methodological framework. International journal of social research methodology 8(1): 19-32.

9. Gough D, Thomas J, Oliver S (2012) Clarifying differences between review designs and methods. Syst Rev 1(1): 28.

10. Locke M, Eccleston S, Ryan CN, Byrnes TJ, Mount C, et al. (2016) Developing a diary program to minimize patient and family postintensive care syndrome. AACN Adv Crit Care 27(2): 212-220.

11. Celik S, Genc G, Kinetli Y, Asiliogli M, Sari M, et al. (2016) Sleep problems, anxiety, depression and fatigue on family members of adult intensive care unit patients. Int J Nurs Pract 22(5): 512-522.

12. Kose I, Zincircioglu C, Ozturk YK, Cakmak M, Guldogan EA, et al. (2016) Factors affecting anxiety and depression symptoms in relatives of intensive care unit patients. J Intensive Care Med 31(9): 611-617.

13. Held N, Mealer M, Clark BJ, Moss M, Sottile PD (2018) Family Member Perspectives and Coping Strategies to Manage Anxiety, Depression, and Acute Stress in the Medical Intensive Care Unit: A Qualitative Study. In A108. American Thoracic Society. American Journal of Respiratory and Critical Care Medicine 197: A2653-A2653.

14. Rusinova K, Kukal J, Simek J, Cerny V (2014) Limited family members/ staff communication in intensive care units in the Czech and Slovak Republics considerably increases anxiety in patients' relatives-the DEPRESS study. BMC psychiatry 14(1): 21.

15. Wolters A, Bouw M, Vogelaar J, Tjan D, van Zanten A, et al. (2015) The Postintensive care syndrome of survivors of critical illness and their families. J Clin Nurs 5-6: 876-879.

16. Hwang DY, Yagoda D, Perrey HM, Currier PF, Tehan TM, et al. (2014) Anxiety and depression symptoms among families of adult intensive care unit survivors immediately following brief length of stay. J Crit Care 29(2): 278-282.

17. Dahlrup B, Ekstrom H, Nordell E, Elmstahl S (2015) Coping as a caregiver: A question of strain and its consequences on life satisfaction and health-related quality of life. Archives of gerontology and geriatrics 61(2): 261-270. 
18. Long AC, Engelberg RA, Downey L, Kross EK, Reinke LF, et al. (2014) Race, income, and education: associations with patient and family ratings of end-of-life care and communication provided by physiciansin-training. J Palliat Med 17(4): 435-447.

19. Lewis CL, Taylor JZ (2017) Impact of prior ICU experience on ICU patient family members psychological distress: A descriptive study. Intensive Crit Care Nurs 43: 129-135.

20. Sottile PD, Lynche Y, Mealer M, Moss M (2014) A24 End of Life and Stressors In The ICU: Resiliency Is Associated with Less Symptoms of Depression, Anxiety, And Improved Satisfaction in Family Members of Intensive Care Unit Patients. American Journal of Respiratory and Critical Care Medicine 189: 1.

21. McPeake J, Devine H, MacTavish P, Fleming L, Crawford R, et al. (2016) Struthers R, Kinsella J, Daniel M, Shaw M, Quasim T. Caregiver strain following critical care discharge: An exploratory evaluation. J Crit Care 35: $180-184$

22. Kao YY, Chen CI, Chen FJ, Lin YH, Perng SJ, et al. (2016) Effects of resourcefulness on sleep disturbances, anxiety, and depressive symptoms in family members of intensive care unit patients. Arch Psychiatr Nurs 30(5): 607-613.

23. Konstanti Z, Gouva M, Dragioti E, Nakos G, Koulouras V (2016) Symptoms of cardiac anxiety in family members of intensive care unit patients. Am J Crit Care 25(5): 448-456.

24. Eifert GH, Thompson RN, Zvolensky MJ, Edwards K, Frazer NL, et al. (2000) The cardiac anxiety questionnaire: development and preliminary validity. Behav Res Ther 38(10): 1039-1053.

25. Wischmeyer PE, Puthucheary Z, San Millan I, Butz D, Grocott MP (2017) Muscle mass and physical recovery in ICU: innovations for targeting of nutrition and exercise. Curr Opin Crit Care 23(4): 269-78.

26. Teng E, Chui H (1987) The modified mini-mental state examination (3MS). Can J Psychiatry 41(2): 114-121.
27. Zigmond AS, Snaith RP (1983) The hospital anxiety and depression scale. Acta Psychiatr Scand 67(6): 361-370.

28. Beck AT, Steer RA, Carbin MG (1988) Psychometric properties of the Beck Depression Inventory: Twenty-five years of evaluation. Clinical psychology review 8(1): 77-100.

29. Beck AT, Ward CH, Mendelson M, Mock J, Erbaugh J (1961) An inventory for measuring depression. Arch Gen Psychiatry 4(6): 561-571.

30. Zung WW (1965) A self-rating depression scale. Arch Gen Psychiatry 12(1): $63-70$.

31. Biggs JT, Wylie LT, Ziegler VE (1978) Validity of the Zung self-rating depression scale. Br J Psychiatry 132(4): 381-385.

32. Zung WW (1971) A rating instrument for anxiety disorders. Psychosomatics 12(6): 371-379.

33. Curtis JR, Treece PD, Nielsen EL, Gold J, Ciechanowski PS, et al. (2016) Randomized trial of communication facilitators to reduce family distress and intensity of end-of-life care. Am J Respir Crit Care Med 193(2): 154162.

34. Mistraletti G, Umbrello M, Mantovani ES, Moroni B, Formenti P, et al. (2017) A family information brochure and dedicated website to improve the ICU experience for patients' relatives: an Italian multicenter beforeand-after study. Intensive Care Med 43(1): 69-79.

35. Kean S, Smith GD (2014) Surviving critical illness: Intensive care and beyond. Journal of clinical nursing 23(5-6): 603-604.

36. Mitchell M, Coombs M, Wetzig K (2017) The provision of family-centered intensive care bereavement support in Australia and New Zealand: results of a cross sectional explorative descriptive survey. Aust Crit Care 30(3): 139-144.

37. Turnbull AE, Hashem MD, Rabiee A, Chessare CM, Needham DM, et al. (2017) Evaluation of a strategy for enrolling the families of critically ill patients in research using limited human resources. PLoS One 12(5): e0177741. 